\title{
Weighted measure algebras and uniform norms
}

\author{
by
}

\author{
S. J. Bhatt and H. V. Dedania (Vallabh Vidyanagar)
}

\begin{abstract}
Let $\omega$ be a weight on an LCA group $G$. Let $M(G, \omega)$ consist of the Radon measures $\mu$ on $G$ such that $\omega \mu$ is a regular complex Borel measure on $G$. It is proved that: (i) $M(G, \omega)$ is regular iff $M(G, \omega)$ has unique uniform norm property (UUNP) iff $L^{1}(G, \omega)$ has UUNP and $G$ is discrete; (ii) $M(G, \omega)$ has a minimum uniform norm iff $L^{1}(G, \omega)$ has UUNP; (iii) $M_{00}(G, \omega)$ is regular iff $M_{00}(G, \omega)$ has UUNP iff $L^{1}(G, \omega)$ has UUNP, where $M_{00}(G, \omega):=\left\{\mu \in M(G, \omega): \widehat{\mu}=0\right.$ on $\left.\Delta(M(G, \omega)) \backslash \Delta\left(L^{1}(G, \omega)\right)\right\}$.
\end{abstract}

1. Introduction. A uniform norm on a Banach algebra $(\mathcal{A},\|\cdot\|)$ is a (not necessarily complete) norm $|\cdot|$ on $\mathcal{A}$ satisfying the square property $\left|a^{2}\right|=|a|^{2}(a \in \mathcal{A})$. A minimum uniform norm on $\mathcal{A}$ is a minimum norm among all uniform norms on $\mathcal{A}$. A Banach algebra $\mathcal{A}$ has the Unique Uniform Norm Property (UUNP) if $\mathcal{A}$ admits exactly one uniform norm [BhDe1]; in this case, the spectral radius $r(\cdot)$ is the only uniform norm on $\mathcal{A}$. The perspective of UUNP in Banach algebras is discussed in [BhDe1] and [BhDe2]. By [BhDe2, Theorem 4.1] and [BhDe3], the Beurling algebra $L^{1}(G, \omega)$ has UUNP if and only if $L^{1}(G, \omega)$ is regular. On the other hand, either $L^{1}(G, \omega)$ has exactly one uniform norm or it has infinitely many uniform norms [BhDe4].

The present note is aimed at investigating UUNP in weighted measure algebra $M(G, \omega)$. In what follows, we briefly discuss preliminaries to fix up the notations.

Throughout let $G$ be an LCA group with the Haar measure $\lambda$. Let $\widehat{G}$ be the dual group of $G$. A generalized character on $G$ is a continuous homomorphism $\alpha: G \rightarrow(\mathbb{C} \backslash\{0\}, \times)$. Let $H(G)$ denote the set of all generalized characters on $G$ equipped with the compact-open topology. For $\alpha, \beta \in H(G)$, define $(\alpha+\beta)(s)=\alpha(s) \beta(s)(s \in G)$. If $G$ is compactly generated, then $H(G)$ is an LCA group [BhDe5]. Let $C_{\mathrm{c}}(G)$ denote the set of all continuous

2000 Mathematics Subject Classification: 43A10, 43A20, 46J05.

Key words and phrases: generalized character, Beurling algebra, weighted measure algebra, Fourier-Stieltjes transform, Laplace transform, minimum uniform norm, unique uniform norm property, commutative semisimple Banach algebra. 
functions on $G$ having compact supports equipped with the inductive limit topology $\tau$. Then $\left(C_{\mathrm{c}}(G), \tau\right)$ is a commutative topological algebra with the convolution product. For $\alpha \in H(G)$, define

$$
\varphi_{\alpha}(f)=\int_{G} f(s) \alpha(s) d \lambda(s) \quad\left(f \in C_{\mathrm{c}}(G)\right) .
$$

Then $\varphi_{\alpha} \in \Delta\left(C_{\mathrm{c}}(G)\right)$, the Gelfand space of $C_{\mathrm{c}}(G)$. Let $T: H(G) \rightarrow$ $\Delta\left(C_{\mathrm{c}}(G)\right), \alpha \mapsto \varphi_{\alpha}$. If $G$ is second countable and compactly generated, then $T$ is a homeomorphism [BhDe5].

By a weight on $G$, we mean a continuous function $\omega: G \rightarrow(0, \infty)$ such that $\omega(s+t) \leq \omega(s) \omega(t)(s, t \in G)$. The associated Beurling algebra is the convolution Banach algebra $L^{1}(G, \omega)$ of all measurable functions $f$ on $G$ satisfying $\|f\|_{\omega}:=\int_{G}|f| \omega d \lambda<\infty$. An element $\alpha \in H(G)$ is an $\omega$-bounded generalized character if $|\alpha(s)| \leq \omega(s)(s \in G)$; let $H(G, \omega)$ denote the set of all such elements of $H(G)$. Let $T_{1}$ be the map $T$ restricted to $H(G, \omega)$. Then $T_{1}$ is a homeomorphism from $H(G, \omega)$ onto $\Delta\left(L^{1}(G, \omega)\right)$ for any LCA group.

2. Weighted measure algebras and UUNP. A Radon measure on $G$ is a continuous linear functional on $C_{\mathrm{c}}(G)$. Let $M_{\text {loc }}(G)$ denote the linear space of all Radon measures on $G$ (see [Da]). Let $L_{\mathrm{loc}}(G)$ denote the space of all locally integrable, measurable functions on $G$. Then clearly $L_{\mathrm{loc}}(G) \subseteq$ $M_{\text {loc }}(G)$. Let $M(G)$ be the convolution Banach algebra of all complex regular Borel measures (necessarily finite) on $G$ with the total variation norm $\|\cdot\|$. For a weight $\omega$ on $G$, define

$$
\begin{aligned}
M(G, \omega) & =\left\{\mu \in M_{\mathrm{loc}}(G): \omega \mu \in M(G)\right\}, \\
C_{0}(G, 1 / \omega) & =\left\{f \in C(G): f / \omega \in C_{0}(G)\right\}, \\
C_{\mathrm{b}}(G, 1 / \omega) & =\left\{f \in C(G): f / \omega \in C_{\mathrm{b}}(G)\right\} .
\end{aligned}
$$

All are Banach spaces; the first one with norm $\|\mu\|_{\omega}:=\|\omega \mu\|$ and the others with norm $\|f\|_{\omega^{-1}, \infty}:=\left\|\omega^{-1} f\right\|_{\infty}$. It is clear that every $\mu \in M(G, \omega)$ is a continuous linear functional on $C_{\mathrm{b}}(G, 1 / \omega)$ by $\langle f, \mu\rangle=\int_{G} f / \omega d \nu$, where $\nu=\omega \mu \in M(G)$. For $\mu \in M(G, \omega)$ and $f \in C_{\mathrm{b}}(G, 1 / \omega)$, define

$$
T_{f}^{\mu}: G \rightarrow \mathbb{C} \quad \text { as } \quad T_{f}^{\mu}(s)=\left\langle\tau_{-s} f, \mu\right\rangle \quad(s \in G) .
$$

Then $T_{f}^{\mu} \in C_{\mathrm{b}}(G, 1 / \omega)$ and $\left\|T_{f}^{\mu}\right\|_{\omega^{-1}, \infty} \leq\|\mu\|_{\omega}\|f\|_{\omega^{-1}, \infty}$. For $\mu, \nu \in M(G, \omega)$, the convolution product $\mu * \nu$ is defined as

$$
\langle f, \mu * \nu\rangle=\left\langle T_{f}^{\mu}, \nu\right\rangle \quad\left(f \in C_{\mathrm{b}}(G, 1 / \omega)\right) .
$$

Then it is a routine verification that $\mu * \nu \in M(G, \omega), \mu * \nu=\nu * \mu$ and $\|\mu * \nu\|_{\omega} \leq\|\mu\|_{\omega}\|\nu\|_{\omega}$. Thus $M(G, \omega)$ is a commutative Banach algebra. The pointmass measure $\delta_{0}$ is the identity of $M(G, \omega)$. The Beurling algebra 
$L^{1}(G, \omega)$ is a closed ideal in $M(G, \omega)$; and by [Gh, Lemma 2.3], $M(G, \omega)$ is exactly the multiplier algebra of $L^{1}(G, \omega)$.

Let $\mu \in M_{\text {loc }}(G)$. Define $D_{\mathcal{L}_{\mu}}=\left\{\alpha \in H(G): \int_{G}|\alpha(s)| d|\mu|(s)<\infty\right\}$. When $D_{\mathcal{L} \mu} \neq \emptyset, \mathcal{L} \mu$ (also denoted by $\left.\widehat{\mu}\right)$, defined as

$$
(\mathcal{L} \mu)(\alpha)=\widehat{\mu}(\alpha)=\int_{G} \alpha(s) d \mu(s) \quad\left(\alpha \in D_{\mathcal{L} \mu}\right),
$$

is the Laplace transform of $\mu$. The following introduces weighted analogues of the classical transforms of harmonic analysis, thereby providing important tools for abelian weighted harmonic analysis.

Proposition 2.1. Let $\mu, \nu \in M_{\mathrm{loc}}(G)$.

(i) $D_{\mathcal{L} \mu} \neq \emptyset$ iff $\mu \in M(G, \omega)$ for some weight $\omega$ on $G$.

(ii) $D_{\mathcal{L} \mu} \cap D_{\mathcal{L} \nu} \neq \emptyset$ iff $\mu, \nu \in M(G, \omega)$ for some weight $\omega$ on $G$. In this case, $\mu * \nu \in M(G, \omega)$ and $D_{\mathcal{L} \mu * \nu} \neq \emptyset$.

(iii) If $\alpha \in D_{\mathcal{L} \mu}$, then $\alpha+\widehat{G} \subseteq D_{\mathcal{L} \mu}$.

(iv) If $\alpha \in D_{\mathcal{L} \mu}$ and if $\mathcal{L} \mu=0$ on $\alpha+\widehat{G}$, then $\mu=0$.

(v) Let $\omega$ be a weight on $G$ and let $\mu \in M(G, \omega)$. Then $H(G, \omega) \subseteq D_{\mathcal{L} \mu}$; the restriction of $\mathcal{L} \mu$ to $H(G, \omega)$ is the generalized Fourier-Stieltjes transform of $\mu$.

(vi) Let $\omega$ be a weight on $G$ and let $f \in L^{1}(G, \omega)$. Then $H(G, \omega) \subseteq$ $D_{\mathcal{L} f}$; the restriction of $\mathcal{L} f$ to $H(G, \omega)$ is the generalized Fourier transform of $f$.

(vii) Let $\omega$ be a weight on $G$ such that $\omega \geq 1$ on $G$ and let $\mu \in M(G, \omega)$. Then $\widehat{G} \subseteq D_{\mathcal{L} \mu}$; the restriction of $\mathcal{L} \mu$ to $\widehat{G}$ is the Fourier-Stieltjes transform of $\mu$.

(viii) Let $\omega$ be a weight on $G$ with $\omega \geq 1$ on $G$ and let $f \in L^{1}(G, \omega)$. Then $\widehat{G} \subseteq D_{\mathcal{L} f}$; the restriction of $\mathcal{L} f$ to $\widehat{G}$ is the Fourier transform of $f$.

Proof. (i) Let $\alpha \in D_{\mathcal{L} \mu}$. Define $\omega(s)=|\alpha(s)|(s \in G)$. Then $\mu \in$ $M(G, \omega)$. Conversely, let $\mu \in M(G, \omega)$ for some weight $\omega$ on $G$. By [BhDe3], $H(G, \omega) \neq \emptyset$. Let $\beta \in H(G, \omega)$. Then $\int_{G}|\beta(s)| d|\mu|(s) \leq \int_{G} \omega(s) d|\mu|(s)<$ $\infty$. Hence $H(G, \omega) \subseteq D_{\mathcal{L} \mu}$. In particular, $D_{\mathcal{L} \mu} \neq \emptyset$.

(ii) Assume that $D_{\mathcal{L} \mu} \cap D_{\mathcal{L} \nu} \neq \emptyset$. Choose $\alpha \in D_{\mathcal{L} \mu} \cap D_{\mathcal{L} \nu}$. Define $\omega(s)=$ $|\alpha(s)|(s \in G)$. Then $\mu, \nu \in M(G, \omega)$. Since $M(G, \omega)$ is a Banach algebra, $\mu * \nu \in M(G, \omega)$. By (i) above, $D_{\mathcal{L} \mu * \nu} \neq \emptyset$. Conversely, let $\mu, \nu \in M(G, \omega)$ for some weight $\omega$ on $G$. Then, as in the proof of $(\mathrm{i}), \emptyset \neq H(G, \omega) \subseteq$ $D_{\mathcal{L} \mu} \cap D_{\mathcal{L} \nu}$.

(iii) For $\alpha \in D_{\mathcal{L}_{\mu}}$ and $\theta \in \widehat{G}, \int_{G}|(\alpha+\theta)(s)| d|\mu|(s)=\int_{G}|\alpha(s)| d|\mu|(s)$ $<\infty$. Hence $\alpha+\widehat{G} \subseteq D_{\mathcal{L} \mu}$. 
(iv) Let $\alpha \in D_{\mathcal{L} \mu}$ be such that $\mathcal{L} \mu=0$ on $\alpha+\widehat{G}$. Since $\alpha \in D_{\mathcal{L} \mu}$, $\alpha \mu \in M(G)$. Now $\mathcal{L}(\alpha \mu)(\theta)=\mathcal{L}(\mu)(\alpha+\theta)=0(\theta \in \widehat{G})$. Hence $\alpha \mu=0$. Since $\alpha(s) \neq 0$ for any $s \in G, \mu=0$.

(v) For $\beta \in H(G, \omega), \int_{G}|\beta(s)| d|\mu|(s) \leq \int_{G} \omega(s) d|\mu|(s)<\infty$.

(vi) This can be proved as (v).

(vii) Let $\theta \in \widehat{G}$. Then $\theta \in H(G)$ and $\int_{G}|\theta(s)| d|\mu|(s) \leq \int_{G} \omega(s) d|\mu|(s)$ $<\infty$. Hence $\widehat{G} \subseteq D_{\mathcal{L} \mu}$.

(viii) This can be proved as in (vii).

Proposition 2.2. Let $\omega$ be a weight on $G$. Then there exists a weight $\widetilde{\omega} \geq 1$ on $G$ such that $M(G, \omega)$ is isometrically isomorphic to $M(G, \widetilde{\omega})$.

Proof. Since $H(G, \omega)$ is non-empty, choose $\alpha \in H(G, \omega)$. Define $\widetilde{\omega}(s)=$ $\omega(s) /|\alpha(s)|(s \in G)$. Then $\widetilde{\omega}$ is a weight and $\widetilde{\omega} \geq 1$ on $G$. Now define $T: M(G, \omega) \rightarrow M(G, \widetilde{\omega})$ as $T(\mu)=\alpha \mu$. Then, for $\mu \in M(G, \omega)$,

$$
\|T(\mu)\|_{\widetilde{\omega}}=\|\alpha \mu\|_{\widetilde{\omega}}=\int_{G} \frac{\omega(s)}{|\alpha(s)|} d|\alpha \mu|(s)=\int_{G} \omega(s) d|\mu|(s)=\|\mu\|_{\omega} .
$$

It is easy to see that $T$ is an algebra isomorphism.

Proposition 2.3. Let $\omega$ be a weight on $G$. Then the following are equivalent:

(i) $M(G, \omega)$ is regular;

(ii) $M(G, \omega)$ has UUNP;

(iii) $L^{1}(G, \omega)$ has UUNP and $G$ is discrete.

Proof. By Proposition 2.2, we can assume that $\omega \geq 1$ on $G$.

(i) $\Rightarrow$ (ii). This is true for all semisimple, commutative Banach algebras.

(ii) $\Rightarrow$ (iii). Since $M(G, \omega)$ is a dense subalgebra of $M(G), M(G)$ has UUNP due to Theorem 2.4 in [BhDe2]. Hence, by [BhDe2, p. 233], $G$ must be discrete. In this case, $L^{1}(G, \omega)=M(G, \omega)$ has UUNP.

(iii) $\Rightarrow\left(\right.$ i). Since $G$ is discrete, $M(G, \omega)=L^{1}(G, \omega)$. By [BhDe2, Theorem 4.1] and [BhDe3], $L^{1}(G, \omega)$ is regular.

The following is the main result of the paper. It compares with the result that $L^{1}(G, \omega)$ has a minimum uniform norm if and only if $L^{1}(G, \omega)$ has UUNP [BhDe4, Theorem 1].

THEOREM 2.4. $M(G, \omega)$ has a minimum uniform norm if and only if $L^{1}(G, \omega)$ has UUNP.

Proof. By Proposition 2.2, we can assume that $\omega \geq 1$ on $G$. Assume that $L^{1}(G, \omega)$ has UUNP. Then it is regular due to [BhDe2, Theorem 4.1]. Since $M(G, \omega)$ is the multiplier algebra of $L^{1}(G, \omega)$ and $\Delta\left(L^{1}(G, \omega)\right) \cong H(G, \omega)$ is a set of uniqueness for $M(G, \omega)$ by Proposition 2.1, it follows from [BhDe2, 
Corollary 6.3] that $|\mu|_{\infty}:=\sup \{|\widehat{\mu}(\alpha)|: \alpha \in H(G, \omega)\}$ is the minimum uniform norm on $M(G, \omega)$.

Conversely, assume that $M(G, \omega)$ has a minimum uniform norm, say $|\cdot|_{0}$. Define $F=\left\{\alpha \in H(G, \omega): \alpha\right.$ is $|\cdot|_{0}$-continuous $\}$. Then $|f|_{0}=\sup \left\{\left|\varphi_{\alpha}(f)\right|\right.$ : $\alpha \in F\}:=|f|_{F}\left(f \in L^{1}(G, \omega)\right)$. We break up the proof into three steps.

SteP I: $|\cdot|_{0}$ is a minimum uniform norm on $L^{1}(G, \omega)$. Let $|\cdot|$ be any uniform norm on $L^{1}(G, \omega)$. Define

$$
|\mu|_{\mathrm{op}}=\sup \left\{|f * \mu|: f \in L^{1}(G, \omega) \text { and }|f| \leq 1\right\} \quad(\mu \in M(G, \omega)) .
$$

Since $L^{1}(G, \omega)$ has a bounded approximate identity, $|\cdot|_{\text {op }}$ is a uniform norm on $M(G, \omega)$ and it is identical to $|\cdot|$ on $L^{1}(G, \omega)$. Since $|\cdot|_{0}$ is a minimum uniform norm on $M(G, \omega),|\cdot|_{0} \leq|\cdot|_{\text {op }}$ on $M(G, \omega)$ and hence $|\cdot|_{0} \leq|\cdot|_{\text {op }}=|\cdot|$ on $L^{1}(G, \omega)$. Thus $|\cdot|_{0}$ is a minimum uniform norm on $L^{1}(G, \omega)$.

SteP II: $F=\widehat{G}+F$. Fix $\theta \in \widehat{G}$. Define $|f|_{\theta+F}=|\theta f|_{F}$ on $L^{1}(G, \omega)$, where $\theta+F:=\{\theta+\alpha: \alpha \in F\}$. Then $|\cdot|_{\theta+F}$ is a uniform norm on $L^{1}(G, \omega)$. Since $|\cdot|_{F}\left(=|\cdot|_{0}\right)$ is the minimum uniform norm on $L^{1}(G, \omega)$, we have

$$
|f|_{F} \leq|f|_{\theta+F} \quad\left(f \in L^{1}(G, \omega)\right) .
$$

This holds for each $\theta \in \widehat{G}$. So $|f|_{\theta+F} \leq|\theta f|_{\bar{\theta}+F}=|f|_{F}$. Hence $\bar{\theta}+F \subseteq F$ by the definition of $F$ and $|\cdot|_{F}=|\cdot|_{0}$. Thus it follows that $F=\widehat{G}+F$.

SteP III: $F=H(G, \omega)$. Suppose this is not the case. Then there exist positive generalized characters $\alpha \in F$ and $\beta \in H(G, \omega) \backslash F$ because $F=$ $\widehat{G}+F$. Choose $t \in G$ such that $\beta(t)<\alpha(t)$. Let $U$ be an open neighbourhood of $t$ such that its closure is compact and $\beta(s)<\alpha(s)(s \in U)$. Take $f=\chi_{U}$. Then $f \in L^{1}(G, \omega)$ because $\omega$ is continuous. Now

$$
\begin{aligned}
|f|_{\beta+\widehat{G}} & =\sup \left\{\left|\varphi_{\beta+\theta}(f)\right|: \theta \in \widehat{G}\right\} \\
& =\sup \left\{\left|\int_{G} f(s) \beta(s) \theta(s) d \lambda(s)\right|: \theta \in \widehat{G}\right\} \\
& \leq \sup \left\{\int_{U} \beta(s)|\theta(s)| d \lambda(s): \theta \in \widehat{G}\right\} \\
& =\int_{U} \beta(s) d \lambda(s)<\int_{U} \alpha(s) d \lambda(s) \\
& \leq \sup \left\{\left|\varphi_{\alpha+\theta}(f)\right|: \theta \in \widehat{G}\right\}=|f|_{\alpha+\widehat{G}} \leq|f|_{F} .
\end{aligned}
$$

Thus $|\cdot|_{\beta+\widehat{G}}$ is a uniform norm and $|f|_{\beta+\widehat{G}}<|f|_{F}$, which is a contradiction because the latter is the minimum uniform norm on $L^{1}(G, \omega)$. Thus $F=$ $H(G, \omega)$.

By Step III, $|f|_{0}=|f|_{F}=|f|_{H(G, \omega)}=r(f)\left(f \in L^{1}(G, \omega)\right)$, where $r(\cdot)$ denotes the spectral radius on $L^{1}(G, \omega)$. Thus the spectral radius is the only uniform norm on $L^{1}(G, \omega)$, and hence $L^{1}(G, \omega)$ has UUNP. 
Example 2.5. For $\alpha \geq 0$, define $\omega_{\alpha}(s)=(1+|s|)^{\alpha}(s \in \mathbb{R})$. Then the Beurling algebra $L^{1}\left(\mathbb{R}, \omega_{\alpha}\right)$ has UUNP. Thus $M\left(\mathbb{R}, \omega_{\alpha}\right)$ has a minimum uniform norm, namely $\mu \mapsto|\widehat{\mu}|_{\infty}$, but $M\left(\mathbb{R}, \omega_{\alpha}\right)$ fails to have UUNP.

Let

$$
\begin{aligned}
M_{0}(G, \omega): & =\left\{\mu \in M(G, \omega): \widehat{\mu} \in C_{0}\left(\Delta\left(L^{1}(G, \omega)\right)\right)\right\} \\
& =\left\{\mu \in M(G, \omega): \widehat{\mu} \in C_{0}(H(G, \omega))\right\}
\end{aligned}
$$

and

$$
\begin{aligned}
M_{00}(G, \omega): & =\left\{\mu \in M(G, \omega): \widehat{\mu}=0 \text { on } \Delta(M(G, \omega)) \backslash \Delta\left(L^{1}(G, \omega)\right)\right\} \\
& =\{\mu \in M(G, \omega): \widehat{\mu}=0 \text { on } \Delta(M(G, \omega)) \backslash H(G, \omega)\} \\
& =\left\{\mu \in M(G, \omega): \widehat{\mu}=0 \text { on } h\left(L^{1}(G, \omega)\right)\right\},
\end{aligned}
$$

where $h\left(L^{1}(G, \omega)\right)$ is the hull of $L^{1}(G, \omega)$ in $M(G, \omega)$. Then both are closed ideals in $M(G, \omega)$ and $L^{1}(G, \omega) \subseteq M_{00}(G, \omega) \subseteq M_{0}(G, \omega)$. These are weighted measure algebra analogues of $M_{0}(G)$ and $M_{00}(G)$ considered in [LN, p. 376]. Our feeling is that $M_{00}(G, \omega)$ is close in spirit to $L^{1}(G, \omega)$, whereas $M_{0}(G, \omega)$ is close to $M(G, \omega)$. The following supports this. It also compares with Proposition 2.3.

Proposition 2.6. Let $\omega$ be a weight on $G$. Then the following are equivalent:

(i) $M_{00}(G, \omega)$ is regular;

(ii) $M_{00}(G, \omega)$ has UUNP;

(iii) $L^{1}(G, \omega)$ has UUNP.

Proof. (i) $\Rightarrow$ (ii). This is true for all semisimple, commutative, Banach algebras.

(ii) $\Rightarrow$ (iii). Let $M_{00}(G, \omega)$ have UUNP. Let $|\cdot|$ be a uniform norm on $L^{1}(G, \omega)$. Define

$$
|\mu|_{\mathrm{op}}=\sup \left\{|f * \mu|: f \in L^{1}(G, \omega) \text { and }|f| \leq 1\right\} \quad\left(\mu \in M_{00}(G, \omega)\right) .
$$

Then $|\cdot|_{\text {op }}$ is a uniform norm on $M_{00}(G, \omega)$ and it is identical to $|\cdot|$ on $L^{1}(G, \omega)$. Since $M_{00}(G, \omega)$ has UUNP, $|\cdot|_{\text {op }}$ is identical to the spectral radius of $M_{00}(G, \omega)$. Since $L^{1}(G, \omega)$ is an ideal in $M_{00}(G, \omega),|\cdot|=|\cdot|_{\text {op }}$ is identical to the spectral radius on $L^{1}(G, \omega)$. Thus $L^{1}(G, \omega)$ has UUNP.

(iii) $\Rightarrow\left(\right.$ i). Assume that $L^{1}(G, \omega)$ has UUNP. By [BhDe2, Theorem 4.1], $L^{1}(G, \omega)$ is regular. Since $\left.L^{1}(G, \omega)\right) \subseteq M_{00}(G, \omega)$ and since $\Delta\left(L^{1}(G, \omega)\right)=$ $\Delta\left(M_{00}(G, \omega)\right), M_{00}(G, \omega)$ is regular.

Conjecture. Motivated by the fact that $M_{0}(G)$ fails to have UUNP [BhDe2, p. 234], we conjecture that $M_{0}(G, \omega)$ has UUNP if and only if $L^{1}(G, \omega)$ has UUNP and $G$ is discrete. 
Acknowledgements. The authors gratefully acknowledge the financial support from the University Grants Commission as a major research project letter No. F.8-9/2004(SR). The authors are also grateful to UGC-SAP-DRS grant No. F.510/5/DRS/2004(SAP-I) provided to the Department of Mathematics, Sardar Patel University.

\section{References}

[BhDe1] S. J. Bhatt and H. V. Dedania, Banach algebras with unique uniform norm, Proc. Amer. Math. Soc. 124 (1996), 579-584.

[BhDe2] - - - Banach algebras with unique uniform norm II, Studia Math. 147 (2001), 211-235.

[BhDe3] - - - A Beurling algebra is semisimple: an elementary proof, Bull. Austral. Math. Soc. 66 (2002), 91-93.

[BhDe4] -, -, Beurling algebras and uniform norms, Studia Math. 160 (2004), 179-183.

[BhDe5] - - - A note on generalized characters, Proc. Indian Acad. Sci. (Math. Sci.) 115 (2005), 437-444.

[Da] H. G. Dales, Banach Algebras and Automatic Continuity, London Math. Soc. Monogr. 24, Clarendon Press, Oxford, 2000.

[Gh] F. Ghahramani, Weighted group algebra as an ideal in its second dual space, Proc. Amer. Math. Soc. 90 (1984), 71-76.

[LN] K. B. Laursen and M. M. Neumann, An Introduction to Local Spectral Theory, London Math. Soc. Monogr. 20, Clarendon Press, Oxford, 2000.

Department of Mathematics

Sardar Patel University

Vallabh Vidyanagar 388120, Gujarat, India

E-mail: subhashbhaib@yahoo.co.in

hvdedania@yahoo.com

Received August 23, 2005

Revised version October 12, 2006 